\title{
Graphite as a Bose Metal
}

\author{
Yakov Kopelevich \\ Instituto de Física “Gleg Wataghin”, Universidade Estadual de Campinas, Unicamp 13083-970, Campinas, São Paulo, Brasil
}

Received on 31 July, 2003.

\begin{abstract}
Although a considerable amount of the research work has been done on graphite, its physical properties are still not well understood, and novel phenomena such as the magnetic-field-driven metal-insulator transition (MIT), the quantum Hall effect, ferromagnetic and superconducting correlations have recently been revealed. Theoretical analysis suggests that the MIT in graphite is the condensed-matter realization of the magnetic catalysis phenomenon known in relativistic theories of $(2+1)$ - dimensional Dirac fermions (DF), i. e. that the applied field opens an insulating gap in the spectrum of DF, associated with the electron-hole pairing. On the other hand, we demonstrate in this paper that a two parameter scaling analysis proposed by Das and Doniach [D. Das and S. Doniach, Phys. Rev. B 64, 134511 (2001)] to characterize the magnetic-field-tuned Bose metal - insulator transition can be well applied to the MIT observed in graphite. We discuss the possibility that the MIT in graphite is associated with the transition between Bose metal and excitonic insulator states.
\end{abstract}

The apparent metal-insulator transition (MIT) in twodimensional (2D) electron (hole) systems which takes place either varying carrier concentration or applying a magnetic field $\mathrm{H}$ has attracted a broad research interest [1]. Recently, a similar MIT driven by a magnetic field applied perpendicular to basal planes has been reported for graphite [2 - 5], and attributed to the metal - excitonic insulator transition [68]. In this paper we demonstrate that the MIT in graphite can also be understood as a Bose metal - insultor transition (BM-IT). In particular, it is found that the two parameter scaling analysis proposed by Das and Doniach [9] to characterize the BM-IT in 2D systems can be well applied to the MIT measured in graphite. We speculate that the MIT in graphite is associated with the transition between Bose metal and excitonic insulator states.

Magnetotransport measurements have been performed on several well-characterized [4,5] quasi-2D highly oriented pyrolitic graphite (HOPG) samples obtained from the Research Institute "Graphite" (Moscow) and the Union Carbide Co. Here, we present the results of the basalplane resistance $R_{b}(H, T)$ measurements obtained on two HOPG samples with the room temperature, zero-field outof-plane/basal-plane resistivity ratio $\rho_{c} / \rho_{b}=5 \times 10^{4}$ and $\rho_{b}(T=300 K)=3 \mu \Omega c m$ and $\rho_{b}(T=300 K)=5 \mu \Omega c m$ for the samples labeled respectively as HOPG-UC and HOPG-3. Low-frequency ( $\mathrm{f}=1 \mathrm{~Hz}$ ) and dc standard fourprobe magnetoresistance measurements were performed on samples with dimensions $4 \times 4 \times 1.2 \mathrm{~mm}^{3}$ (HOPG-3) and $5 \times 5 \times 1 \mathrm{~mm}^{3}$ (HOPG-UC) in the temperature interval $2 K \leq T \leq 300 K$ using different 9 T-magnet He-cryostats. For the measurements, silver past electrodes were placed on the sample surface, while the resistivity values were obtained in a geometry with an uniform current distribution through the sample cross section. All resistance measure- ments were performed in the Ohmic regime in both $H \| \mathrm{c}$ axis and $H \perp \mathrm{c}$-axis applied magnetic field configurations.

The transition from metallic- $\left(d R_{b} / d T>0\right)$ to insulator-like $\left(d R_{b} / d T<0\right)$ behavior of the basal-plane resistance $R_{b}(T, H)$ driven by a magnetic field applied perpendicular to the graphene planes has been observed for all studied graphite samples. Fig. 1 presents $R_{b}(T, H)$ measured for the HOPG-UC sample. As can be seen from Fig. 1, $R_{b}(T)$ has a metallic character at zero or low enough fields. As the applied field exceeds $H \sim 0.5 \ldots 1 \mathrm{kOe}, R_{b}(T)$ becomes insulating-like, suggesting the occurrence of MIT driven by the magnetic field. Fig. 1 shows that $R_{b}(T)$ goes through a shallow minimum at the field-dependent temperature $T_{\min }\left(H>H_{c}\right)$, where $H_{c}$ is a threshold field below which the metallic state of graphite is preserved, and Fig. 2 gives a detailed view of the resistance minimum. It has been demonstrated $[5,10]$ that the magnetic field component perpendicular to basal graphite planes solely drives the MIT imposing certain constrains on theoretical approaches to the MIT.

The characteristic feature of the band structure of a single graphene layer is that there are two isolated points in the first Brillouin zone where the band dispersion is linear $E(k)=\hbar v|k|\left(v=v_{F} \sim 10^{6} \mathrm{~m} / \mathrm{s}\right.$ is the Fermi velocity), so that the electronic states can be described in terms of Dirac equations in two dimensions similar to, for instance, quasiparticles near the gap nodes in superconducting cuprates. The theoretical analysis [6 - 8] suggests that the MIT in graphite is the condensed-matter realization of the magnetic catalysis (MC) phenomenon known in relativistic theories of $(2+1)$ - dimensional Dirac fermions. According to the theory, the magnetic field applied perpendicular to the graphene planes opens an insulating gap in the spectrum of Dirac fermions, associated with an electron-hole pairing, leading 


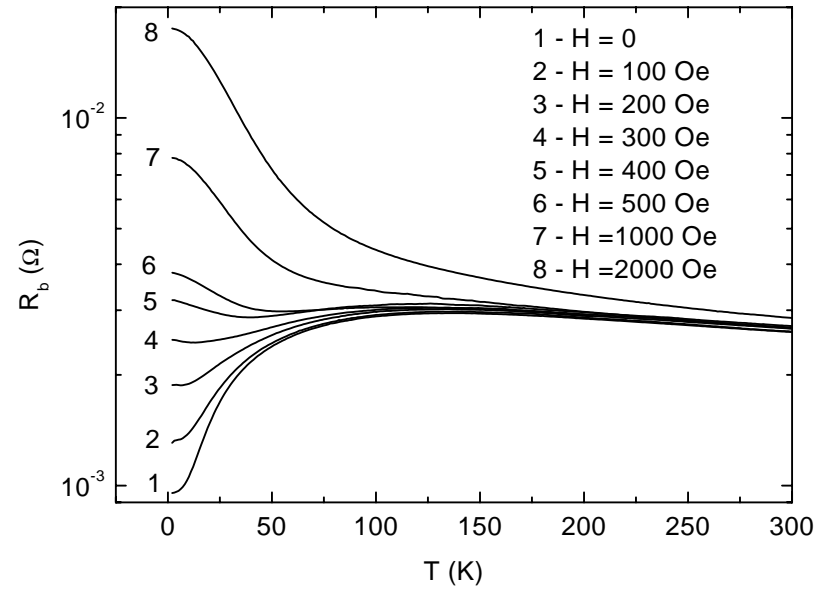

Figure 1. Basal-plane resistance $R_{b}(T, H)$ measured for the sample HOPG-UC at various applied magnetic fields $\mathrm{H} ; \mathrm{H}$ is always (Figs.1-4) applied perpendicular to graphene planes.

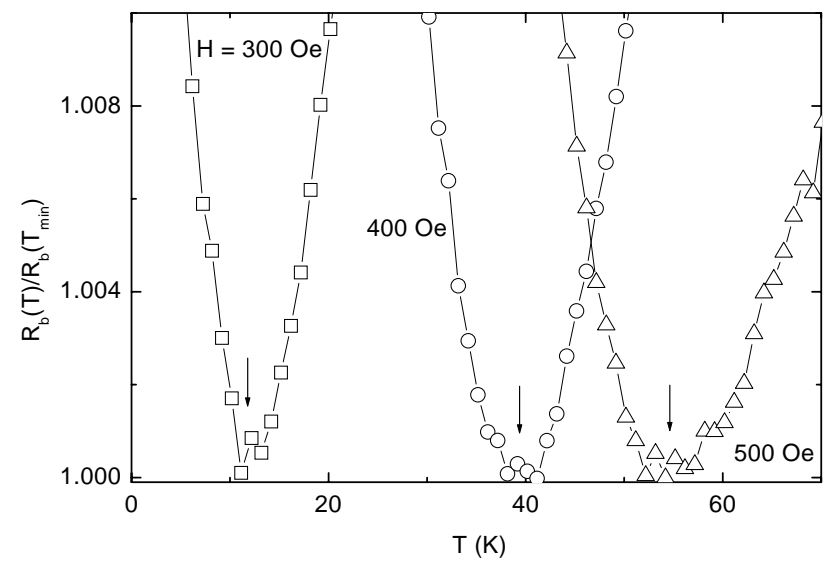

Figure 2. Reduced basal-plane resistance $R_{b}(T) / R_{b}\left(T_{\min }\right)$ measured for the sample HOPG-UC with $\mathrm{H}=300$ Oe $(\square), \mathrm{H}=$ $400 \mathrm{Oe}(\circ), \mathrm{H}=500 \mathrm{Oe}(\triangle)$. Arrows indicate $T_{\min }(H)$, the field-dependent temperature which separates insulating-like $(T<$ $\left.T_{\min }\right)$ and metallic-like $\left(T>T_{\min }\right)$ states.

to the excitonic insulator state below a transition temperature $T_{c e}(H)$ which can be identified with the $T_{\min }(H)$. As shown in Refs. [5, 10], $T_{\min }(H)$ in the vicinity of $H_{c}$ can be well described by theoretically predicted dependencies $T_{\min }(H) \sim\left(H-H_{c}\right)^{1 / 2}$ [6] or $T_{\min }(H) \sim$ $\left[1-\left(H_{c} / H\right)^{2}\right] H^{1 / 2}[8]$, indeed. The second expression for the $T_{\min }(H)$ corresponds to the formula (64) of Ref. [8], $T_{c e} \sim\left(1-\nu_{b}^{2}\right) H^{1 / 2}$, obtained within the MC theory, where $\nu_{b}=2 \pi c n_{2 D} / N_{f}|e H| \equiv H_{c} / H$ is the filling factor, $N_{f}$ is the number of fermion species $\left(N_{f}=2\right.$ for graphite), and $n_{2 D}$ is the $2 \mathrm{D}$ carrier density. We note a two orders of magnitude difference between the predicted value for $\mu_{0} H_{c} \sim 2.5 \mathrm{~T}\left(n_{2 D}=n_{3 D} d \sim 10^{11} \mathrm{~cm}^{-2}\right.$, $n_{3 D} \sim 3 \times 10^{18} \mathrm{~cm}^{-3}$ and $\mathrm{d}=3.35 \AA$, the distance between graphene planes) [8] and experimental value $\mu_{0} H_{c}=$ $0.02 \cdots 0.04 \mathrm{~T}$. The discrepancy can be understood, however, assuming that the Coulomb coupling given by a dimensionless parameter $g=2 \pi e^{2} / \epsilon_{0} v\left(\epsilon_{0}\right.$ is the dielectric constant) drives the system very close to the excitonic instability [6,7]. In this case, the threshold field $H_{c}$ can be well below the estimated value of $2.5 \mathrm{~T}$. The above analysis together with the experimental evidence that only "orbital" effects drive the MIT [5, 10] support the theoretical expectations of the field-induced excitonic insulator state in graphite.

At the same time, our $R_{b}(T, H)$ data resemble very much the resistance behavior occurring in superconducting thin films (see, e. g., Ref. [11]). It has been shown in Refs. [3, 5] that the scaling analysis of the magnetic-fieldinduced superconductor-insulator quantum phase transition (SIT) can be equally well applied to the MIT observed in graphite. According to the scaling theory [12], the resistance in the critical regime of the zero-temperature SIT is given by the equation $R(\delta, T)=R_{c r} f\left(|\delta| / T^{1 / z \nu}\right)$, where $R_{c r}$ is the resistance at the transition, $f\left(|\delta| / T^{1 / z \nu}\right)$ a scaling function such that $\mathrm{f}(0)=1 ; z$ and $\nu$ are critical exponents, and $\delta=H-H_{c r}$ the deviation of a variable parameter from its critical value. However, at low enough temperatures, where the resistance $R_{b}(T)$ saturates, a clear deviation from the scaling takes place in both superconducting films [11] and graphite $[3,5]$. More recently, it has been suggested that the SIT measured, e. g., in Mo-Ge films [11] is in fact a Bose metal - insulator transition, and a two parameter scaling formula $R T^{1+2 / z} / \delta^{2 \beta}=f\left(\delta / T^{1 / z \nu}\right)$, has been proposed to characterize it [9], where $\beta=\nu(z+2) / 2$. This analysis implies the existence of a non-superfluid liquid of Cooper pairs (Bose metal) in the zero-temperature limit. In Fig. 4 we use this approach to analyze the data obtained for HOPG3 sample (see Fig. 3). As Fig. 4 illustrates, this new scaling formula works very well in the whole studied temperature interval, taking $H_{c r}=1140 \mathrm{Oe}, z=1$, and $\nu=2 / 3$.

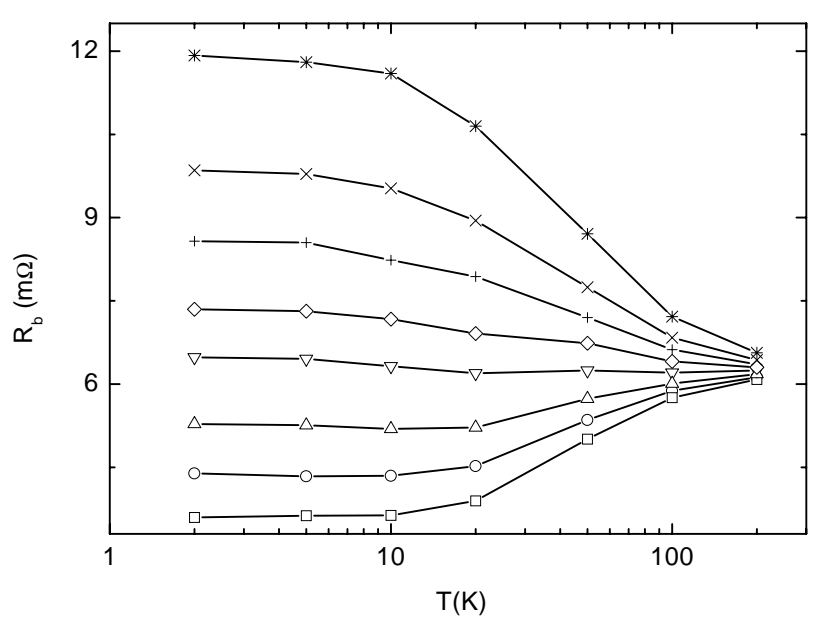

Figure 3. Basal-plane resistance $R_{b}(T, H)$ measured for the sample HOPG-3 at various magnetic fields (from bottom to the top: $\mathrm{H}$ $=500,700,900,1140,1300,1500,1700$, and $2000 \mathrm{Oe}$ ). 


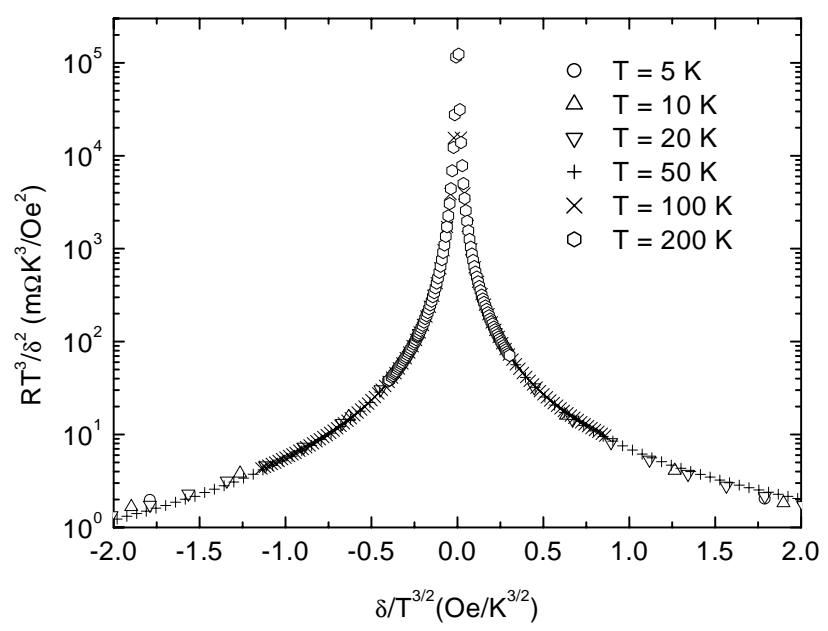

Figure 4. Scaling analysis of the basal-plane resistance $R(T, H) \equiv$ $R_{b}(T, H)$ data presented in Fig. 3, assuming the magnetic-fieldinduced Bose metal - insulator transition occurrence; $\delta=H-H_{c r}$, $H_{c r}=1140 \mathrm{Oe}, z=1$, and $\nu=2 / 3$ (see also text).

The obtained results suggest an interesting possibility, namely an occurrence of the magnetic-field-driven Bose metal - excitonic insulator transition (BM-EIT). We assume that the sample nano-scale inhomogeneities [13] play a crucial role in this phenomenon, leading to co-existing metallic and excitonic insulator regions in graphite at $\mathrm{H}=0[6$, 7]. Then, the Cooper pairs can be formed as a result of the electron-exciton interaction during a tunneling event of electrons from metallic regions into the insulating ones [14, 15]. Notice, that $R_{b}(T)$ at $\mathrm{H}=0$ can be best described by the equation $R_{b}(T)=R_{0}+R_{1} \exp \left(-E_{a} / k_{B} T\right)$, where $E_{a} \approx 35 \mathrm{~K}$ is the effective activation energy [5]. The thermally-activated metallic behavior of $R_{b}(T)$ is consistent with the formation of the superconducting gap in the single particle spectrum, indeed. On the other hand, the high enough applied field opens the excitonic gap in most of the sample and thus drives the BM - EIT.
This work has been supported by FAPESP and CNPq. The author is grateful to D. V. Khveshchenko, S. Doniach, I. A. Shovkovy, P. Esquinazi, and I. A. Luk'yanchuk for a discussion, and J. H. S. Torres and R. R. da Silva for an assistance.

\section{References}

[1] E. Abrahams, S. V. Kravchenko, and M. P. Sarachik, Rev. Mod. Phys. 73, 251 (2001).

[2] Y. Kopelevich, V. V. Lemanov, S. Moehlecke, and J. H. S. Torres, Phys. Solid State 41, 1959 (1999).

[3] H. Kempa, P. Esquinazi and Y. Kopelevich, Phys. Rev. B 65, 241101(R) (2002).

[4] Y. Kopelevich et al., Phys. Rev. Lett. 90, 156402 (2003).

[5] Y. Kopelevich et al., in: Studies of High Temperature Superconductors, ed. by A. Narlikar, Vol. 45, 59 (2003); condmat/0209442.

[6] D. V. Khveshchenko, Phys. Rev. Lett. 87, 206401 (2001).

[7] D. V. Khveshchenko, Phys. Rev. Lett. 87, 246802 (2001).

[8] E. V. Gorbar, V. P. Gusynin, V. A. Miransky, and I. A. Shovkovy, Phys. Rev. B 66, 045108 (2002).

[9] D. Das and S. Doniach, Phys. Rev. B 64, 134511 (2001).

[10] H. Kempa, H. C. Semmelhack, P. Esquinazi, and Y. Kopelevich, Solid State Commun. 125, 1 (2003).

[11] N. Mason and A. Kapitulnik, Phys. Rev. Lett. 82, 5341 (1999).

[12] M. P. A. Fisher, Phys. Rev. Lett. 65, 923 (1990).

[13] Unpublished.

[14] D. Allender, J. Bray, and J. Bardeen, Phys. Rev. B 7, 1020 (1973).

[15] In: High-Temperature Superconductivity, ed. by V. L. Ginzburg and D. A. Kirzhnits, 1982 Consultants Bureau, New York, Division of Plenum Publishing Co. 